\title{
GiggleBat: Enhancing Playing and Outdoor Culture in Australian Children
}

\author{
Omar Mubin, Mauricio Novoa, Joshua Forrester \\ School of Computing, Engineering and \\ Mathematics \\ Western Sydney University \\ Australia \\ o.mubin@westernsydney.edu.au
}

\author{
Rahat lqbal \\ School of Computing, Electronics and \\ Maths, Coventry University \\ CV1 2JH \\ UK \\ r.iqbal@coventry.ac.uk
}

\begin{abstract}
This paper reports on an ongoing design-based research project aiming to improve the physical and social development of children between the ages of 3-5 with tangible interactive sport based play. The project pursues design, evaluation and implementation of an interactive sports toy: GiggleBat that merges benefits of co-design, $\mathrm{HCl}$ and user-centred design (assisted by Arduino sensor technology and 3D Prototyping), together with current Australian playing and sporting culture. The final add-on product serves from established rules and adds value to games of cricket, and potentially tennis and golf by enhancing prompting, information and feedback. We report on our design experience of GiggleBat and evaluation results from a series of focus groups with parents and an observation session with children. We conclude with a future road map for the product.
\end{abstract}

Sports; Play and Learn; Arduino; Children; Cricket; Exergames

\section{INTRODUCTION}

Digital technology has become a major part of everyday life; its rise has provided society with the benefits of comfort and access on a global scale. In Australia, children of all ages use variants of technology for social connections, education and learning, and entertainment such as gaming and television (of Health and Ageing 2010). With technology becoming widely accessible and affordable, designers must look into creative solutions to avoid children getting trapped in an over-indulgence of technology (Lu et al. 2011). Furthermore, children have differing needs to adults, and a large focus should be placed on social interaction (Bekker et al. 2008). Unfortunately, many of the digital applications of technology have led to a lack of social learning within children's education and early development both at home and school. As a result, children risk becoming sedentary through inactive forms of play and learning found in applications and games accessible through digital technology (Attewell et al. 2003). Research into the study of population patterns in Australia further suggests increasing use of technology may result in a higher chance of obesity (Olds et al. 2010).

By now, there is abundant research available that testifies there is an obesity epidemic in the developed and developing world. Data related to child obesity (Wake et al. 2007; of Health and Ageing 2010) show Australian children are increasingly inactive or confined to restrictive spaces for longer periods of time because their parents need to work longer hours or there is less mobility for children in the busy city life (e.g. travel and commuting), or they are longer hours in isolation. Eventually a sedentary life becomes easily addictive (Cassell and Ryokai 2001). That inactivity is reinforced by a number of devices and accessories that exercise the mind over movement, physical activity and community living. In order to overcome static and sedentary behaviour besides regular outdoor games, the importance of sports in childhood development is being stressed. This is evident particularly in pre-school children as it can ensure their longevity in sports involvement (Henrique et al. 2015), reduce the risk of obesity (Dietz and Gortmaker 2001) and promote social connections with peers and parents (Chase and Machida 2011). Therefore sport can be an effective medium to resolve the issue of sedentary behaviour.

We attempted to align and support our research goals with existing theories on child behavior. Our primary research aim of supporting active and social interaction of children with others (including parents) lies in the heart of Vgotsky's theory of zone 
of proximal development (Vygotsky and Wollock 1997). In addition tangible and hands-on activities to facilitate child learning have been advocated for a while in particular through Seymour Papert (Papert and Harel 1991). Therefore the motivation of this project developed from the need to advance on the social development of children between the ages 3 to 5 by encouraging physical and sportive play. In summary our design statement was: To enhance social activity in Australian children while promoting sportive play and enjoyment through a tangible artifact (toy).

\subsection{Background}

A number of sub-topics within Human Computer Interaction $(\mathrm{HCl})$ have contributed towards the well being, learning and physical development of children and teenagers; namely: Interaction Design for children (Avontuur et al. 2014), Mobile and Persuasive Technologies (De Oliveira and Oliver 2008), Sensor based technology (Anlauff et al. 2010), Wearables (Hendrix et al. 2008; Bekker and Eggen 2008), $\mathrm{HCl}$ for Sports or Exergames (Toprak et al. 2012; Mueller et al. 2015), Robots (Mubin et al. 2013), etc. A quick survey of the $\mathrm{HCl}$ field reveals a number of research ventures promoting outdoor play and activity through a merger of digital technology into the physical world by using tangible artifacts infused with mobile devices, electronics or other forms of intelligent products. Head up games (HUG's) (Soute et al. 2010) is one such paradigm advocating mobile based pervasive games for facilitating social context and communication amongst children. Typically in existing HUG's, popular and traditional games are adapted by incorporating digital information within them.

Similarly, persuasive technologies have been utilised to inculcate technology driven behavioral changes in users and promote fitness either through exercise or nutritional adoptions. One such example is the use of mobile apps to facilitate the wellbeing of teenagers (Toscos et al. 2006). $\mathrm{HCl}$ for sports (Mueller et al. 2013) is yet another upcoming research track comprising of various elements including exertion games, virtual play, etc. that through novel forms of technology aim to not only provide entertainment but also to support the fitness requirements of adults in various sports based activities such as jogging (Mueller and Muirhead 2015) and basketball (Bogdanovych and Stanton 2015). However to the best of our knowledge we do not find extensive treatment on the combination of Interaction Design for children and $\mathrm{HCl}$ for Sports, despite a growing recognition of the value exergames could have in keeping adults (Nitz et al. 2010) and children fit (Daley 2009). A couple of notable exceptions to research being conducted in the area of exergames for children are (Bekker and Eggen 2008; Jensen et al. 2013); but the design case and product in (Bekker and Eggen 2008) was reported as a lowfidelity prototype, its applicability to the Australian context is questionable and future research within the project has stayed away from sports based activities (Sturm et al. 2011). On the other hand the focus on using technology to teach football in (Jensen et al. 2013) has been on teenagers. In sum, Pervasive games for children revolve around existing physical (mostly active) games but not sports; whereas $\mathrm{HCl}$ for sports has mostly concentrated on the adult or teenage user group. Additionally many design concepts do not provide an extended range of physical and educational features that remain relevant as a child develops, becoming obsolete either through outgrowing the toy or simply losing interest. There is an underlying need for choice and customisation when children play as they can quickly become disinterested in repetitive actions (Toering et al. 2010). Therefore we wished to support the outdoor play and social development of young children through $\mathrm{HCl} /$ technology advanced sporting toys/artifacts which would allow for customised play in the context of the Australian culture.

\subsection{Sporting culture in Australia}

The importance of sport in Australian culture and society is well established, as is the role of sport in unifying Australians (Rowe 2013). Love for sports is considered an integral component of an Australian's identity with the main sports being cricket, Australian rules football, rugby union, rugby league and association football. Therefore, there is a very strong infrastructure in place in schools, clubs and the community across Australian suburbs to support and facilitate sports based activities for children (Australia 2015). Australian sporting culture is seen as a defining characteristic for its population identity. Only recently it has attracted expert interest as a critical contribution to social capital development (Zakus et al. 2009). However similar to other nations and cultures, social capital development has been progressively declining. There is a reverse relationship between the value of social networks, represented in Australia by its sport culture, and the growth of facilities brought about by technology that diminish the need for collective action, reciprocity and information flows. Famously, Putman (Putnam 2001) studied this syndrome deeply and stated that there is a risk of collapse of community activities and collaboration. Grass roots games such as bowling in America, show a plummeting decline and impoverishment of community connections that start with reduced group play, an increasing number of players bowling alone, and finally adults and children 
ensuing isolation at home once the incentive of communication and social capital disappears.

This project uses digital technologies to support a strategy to reduce isolation and obesity risk in the population while capitalizing on the characteristics of the Australian identity represented by the sport of cricket. Outcomes of that exercise aim to be modulated, replicated and transported to other ares of Australian culture by serving as a means to support social capital development and physical activity. In sum, in order to address the target market of Australian children and their parents we focused our design rationale towards sports based interactive toys. Our design research rationale was to add value to habit currently in use in sport culture, schools, playground and community infrastructure that can easily transport into home and private activity scenarios. With this, the aim was to facilitate a strategy that recovers individual play to strengthen reciprocity and information flows in a movement to enhance feeling of belonging, collective and community action.

\subsection{Methodology}

Our design methodology was initially based on an audit of the state of art technologies followed by a series of qualitative studies with parents of pre-school children in order to attain iterative and comprehensive feedback on our concepts. One dilemma in current research in Interaction Design for children is to determine the extent to which parents should be involved in the design process, particularly as such a venture could lead to a better product. The importance of parents, particularly mothers as a key partner for children in play has been advocated through the Attachment Theory (Wood et al. 2003) and in $\mathrm{HCl}$ (Mubin et al. 2015; Robins et al. 2008). Furthermore we wished to involve children as testers (Druin 2002) in a formative evaluation of our final prototype. Therefore the mothers were involved in the initial design stages and the children were integrated in the final evaluation. The research methodology was approved by the Human Ethics Office of our university. We now report on the design process, implementation using aspects from both Industrial Design and $\mathrm{HCl}$ and a number of iterative evaluations of the GiggleBat product. The evaluations were conducted as a series of three focus groups with mothers and an observation session of their pre-school aged children from an urban region in Australia.

\section{GIGGLEBAT: DESIGN PROCESS}

Primarily based on secondary research (summarised in the previous section) and a larger understanding of the requirements of Australian culture

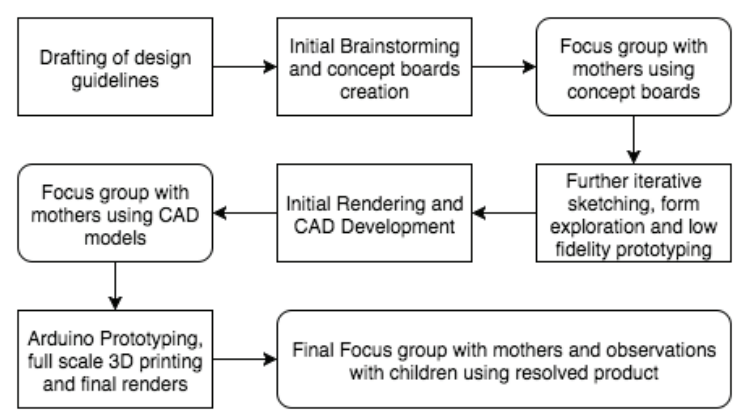

Figure 1: Flow chart of design process

we drew up a list of initial design guidelines that we wished to incorporate in our design concepts. The aspects that the guidelines focused on included: building social relationships with both parents and peers, balancing the presence of digital features and their incorporation in the physical world, to be able to utilise one of the popular sports in Australia, ensure a certain degree of customisation such that it aids in transition between preschool and primary school, encouraging outdoor play, and the toy ultimately being easy to setup and play. An iterative usercentered design process was followed to envision the conceptualisation and prototyping of our product. The process is summarised in a flow chart diagram (see Figure 1).

\subsection{Brainstorming and Concept Development}

The design commenced with an initial screening by the research team (which comprised of $\mathrm{HCl}$ and Design experts based in Australia) of the possible opportunities for the improvement of social and physical development of children. This was achieved through the understanding of different semantics associated with child play in-line with our design guidelines. The visualisation was then converted into a semantic cloud to better understand the world of a modern child. Many of the semantics for children between the ages of 3 to 5 were based on the principles of the sensory system, including sports, colours, music, materials, emotions and imagination. Thereafter, quick and dirty sketching of potential concepts was carried out with a focus on interaction using elements of $\mathrm{HCl}$ and electronics. A large emphasis for the initial sketches was also to communicate a sense of community through social interactions, and physical activity through the use of sport or movement. Some of the concepts were examples of intelligent cricket bats and tennis racquets, portable sports stations, etc. A sample sketch of the portable central sports station kit is provided (see Figure 2). This concept featured holders for parts, stumps for cricket, and a back cutout for a soccer goal. Utilising the Questions, Options 


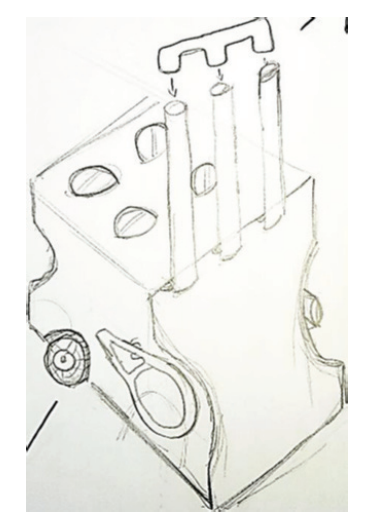

Figure 2: Portable Sports Station

and Criteria Technique (MacLean et al. 1991) and our suggested design guidelines a revised concept was visualised that utilised many features from the earlier brainstormed concepts. We termed this concept as "Gigglebat". In summary, the intended and proposed main design features of Gigglebat were a universal handle that plugs into different sporting equipment (in particular cricket and tennis), provides voice feedback while playing, and lights up when hitting a ball (with variations depending on the quality of the shot). The concept was further articulated through sketching (see Figure 3 ) and with the drafting of a visual and rendered concept board (see Figure 4).

\subsection{Focus Group 1}

In order to attain feedback on our concept and to better understand the target group a focus group was conducted with 7 mothers of pre-school children (each mother currently had at least 1 child of preschool age; i.e. 3 to 5 years old). The initial part of the focus group concentrated on determining the range of play and sports based activities at home and school of the participant's children and also on the requirements of toys and sporting equipment. The second part of the focus group revolved around acquiring feedback on the concept through a discussion of the concept board. The mothers stated that they had a preference for products that required minimal supervision due to the lack of time to spare between work, chores, and parenting. This confirmed our guideline that there is a need for a toy that takes minimal effort to set up and can ultimately be used by children without the constant supervision of parents or guardians. However the mothers agreed that a balance was required between supervised and nonsupervised play. According to the mothers, some of the desired features in interactive sports based toys included durability, gender equality, the ability to share the same toy amongst siblings, the possibility to enhance group play, social and physical activity, etc. Additional feedback on GiggleBat revealed some interesting insights which we now summarise. In general the concept was appreciated as one mother commented: "It's like a Wii for outdoors, but not as expensive". The mothers stressed the importance of scalability and customisation: "Make it possible for different aged children to play together" and "Can the sweet spot be altered for smaller children". Most mothers were happy to see a variety of skills comprised both individually and socially in GiggleBat such as gross and fine motor skills, sharing, confidence and hand-eye coordination.

\subsection{Low fidelity Prototyping, initial CAD models and electronics}

Using the results from the first focus group further development of GiggleBat commenced; including preliminary CAD modelling, resolution of a technical package and a finalised solution. The 3D models (see Figure 5) allowed a visual representation of the proposed concepts and consequent realisation of the technical package. In particular one of the main design challenges was to address the placement of the electronics package within Gigglebat. The underlying technology within GiggleBat focused on the development of a modular handle (ultimately attachable to any compatible bat or racquet) and the sensory system of the bat or racquet. The modularity of the handle would not only potentially increase the usability of the GiggleBat but also enabled to minimise the costs of the expensive electronics (battery, microprocessor, etc) as they could be housed within one common handle; whereas the bats and racquets could house the cheaper parts such as the simple LED's.

Sketches were drawn to understand the technical specifications in a more elaborate fashion (see Figure 6). A number of attachment mechanisms of handle to bat were rendered (see Figure 5) and 3D prototyped but were left unresolved pending further discussion with the mothers in an upcoming Focus group. Also at this juncture we began experimenting with the Arduino platform to realise our design prototype and to simulate our main features of: audio output (praise or encouraging feedback and to ensure turn taking and the possibility of using a micro SD card to play customised sounds), haptic output (vibration when a shot was hit), visual output (LED's in the bat to indicate impact and quality of impact), level customisation (a simple three way-toggle switch situated on the top of the handle allowing to alternate between an off mode and easy and difficult levels), etc. A number of open circuits (see Figure 8 ) were created for demo purposes prior to incorporating them within the handle and/or bat. 

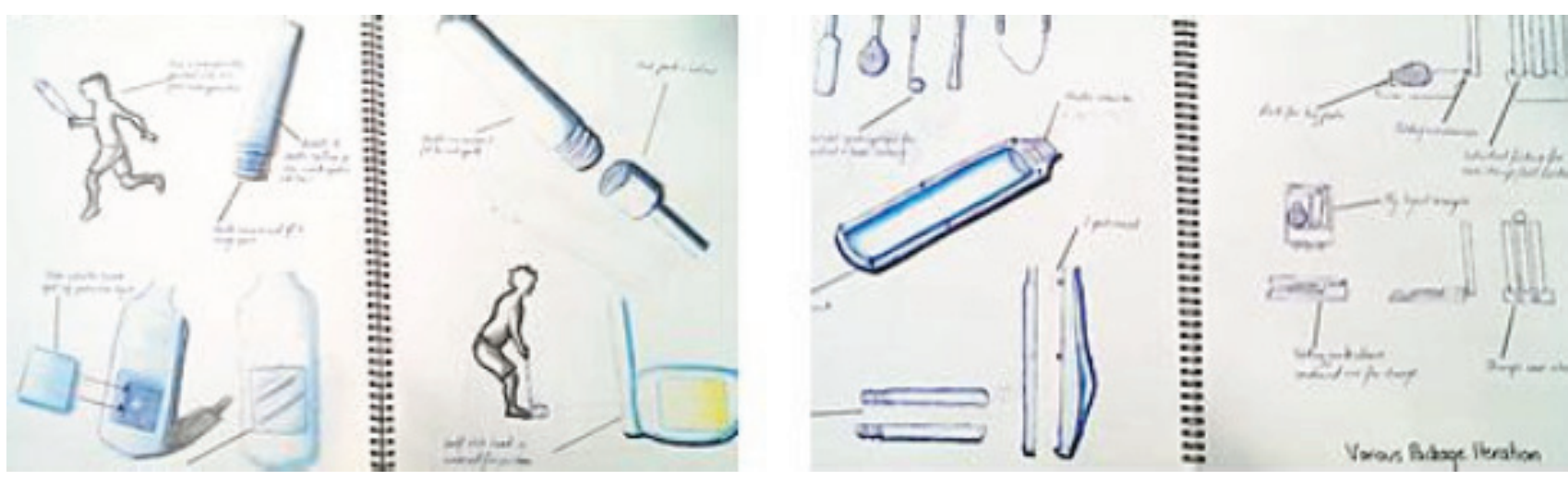

Figure 3: Further sketching of Gigglebat

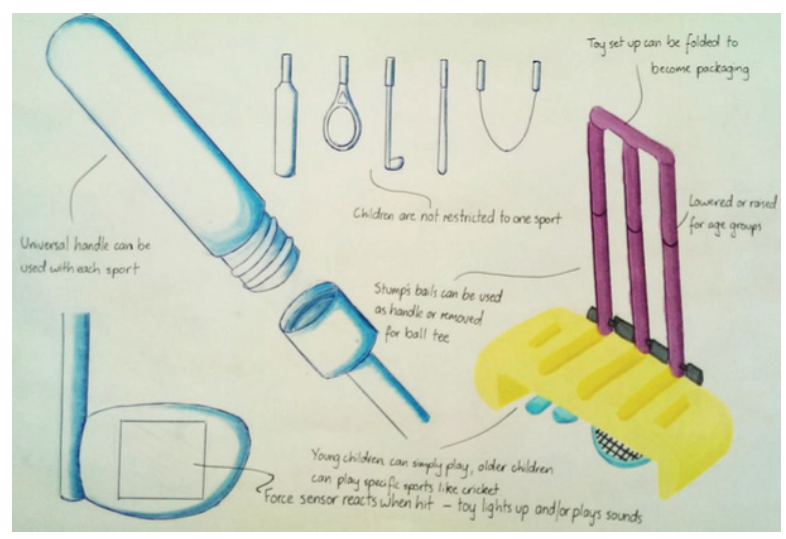

Figure 4: Concept board of Gigglebat

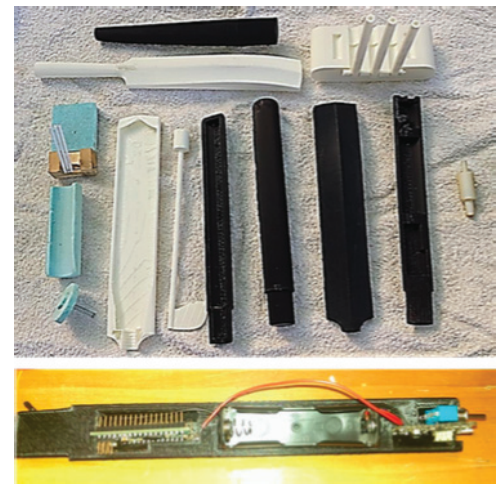

Figure 5: Initial 3D models of bat, handle and casing for technical package

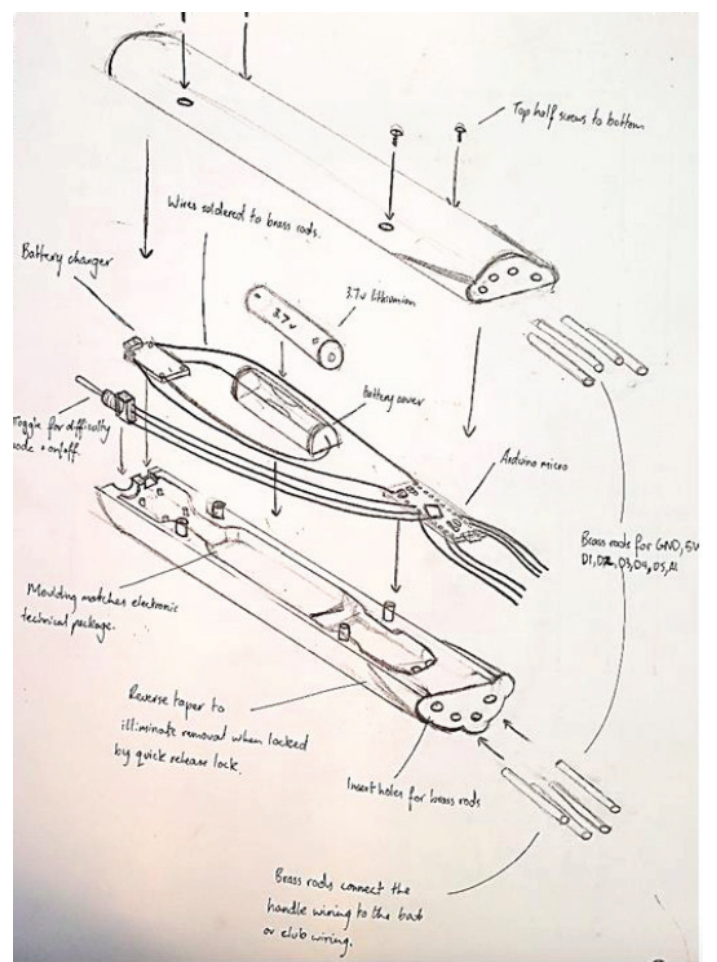

Figure 6: Sketches indicating underlying electronics in Handle

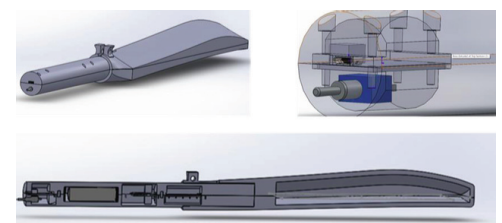

Figure 7: Initial renders showing clamp attachment between handle and bat 


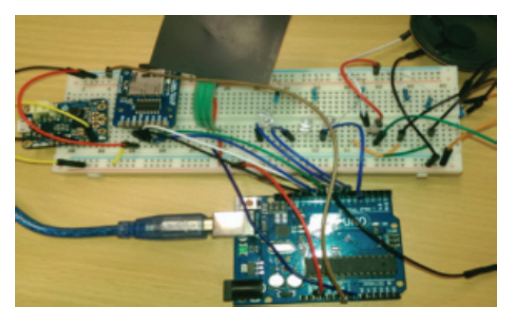

Figure 8: Open circuit showing main features of the GiggleBat

\subsection{Focus Group 2}

A second focus group was conducted using the initial 3D models, visual renders and open Arduino circuits as representation of the GiggleBat concept. 7 mothers participated in this focus group of which 2 were not part of the first focus group. Therefore the two new mothers were brought up to speed with regards to our research aims and the GiggleBat concept. The primary purpose of this focus group was to attain detailed and in-depth feedback on the proposed features of GiggleBat and consequently orient future design work on the concept. Aspects discussed included: desirable features of GiggleBat, possible attachment mechanisms of handle and bat, scope of use of the GiggleBat, possible choices of audio feedback from GiggleBat, etc.

In general the concept was well received with the mothers appreciating the level of customisation provided by the product. For example, the three way toggle switch provided a possibility of the bat being used by children of various age groups: "I know you're aiming for 3-5 but I think my child would find plenty of use well past 5". A number of standard alternatives (as in most commercial toys for preschool children) for the attachment mechanism were discussed including: a quick release clamp, threaded screw, bayonet mount, etc. The most favoured handle attachment method was a quick release clamp, similar to those seen on kick scooter handle bars as it was considered easy for children to manipulate. A significant part of the discussion concentrated on contemplating various alternatives for the audio feedback. The mothers stressed the importance of ensuring that the product had enough variance in speech so that it did not repeat itself. They were uninterested in hearing generic sounds such as "Try again" and generally preferred the same underlying meaning, but rather in a more expressed example; as one mother suggested "That was so close, try watching the ball closer". This example highlights the critical element of providing targeted feedback rather than aimless feedback. Several mothers also agreed that GiggleBat could be an effective tool to facilitate turn taking and sharing through its audio instructions as they felt the children might adhere to it. In addition 4 out of 7 mothers were very keen to introduce trademark phrases from famous Australian cricket commentators such as Richie Benaud, Bill Lawry, etc as part of the audio capability of GiggleBat. Lastly, the modularity of the handle was acknowledged, as all mothers realised that quickly switching the handle to another sports instrument would keep their children interested without feeling bored. In summary, customisation within the GiggleBat design features was deemed as an important aspect.

\subsection{Product Realisation and Final Evaluation}

Using the results from the focus group, product development progressed further with specific attention paid to the design of the clamp attachment. A 3D model of the quick release attachment method was created (see Figure 9) to test the holding strength before the final product was produced. Thereafter using an in-house Objet 3D Printer full scale models of a cricket bat, tennis racquet and their associated modular handle were produced (see Figure 10). The handle designed for the final concept was a universal design that was able to fit into different clubs, racquets and bats, and was made from a polypropylene homopolymer. The bat and racquet pieces however, were manufactured with a polypropylene copolymer. The homopolymer used for the handle was stiffer to enable it to take force without deforming, while the copolymer was more durable in order to withstand constant impact from a ball.

The electronic circuits were finalised and housed within the bat. A summary of the main components (see Figure 11) and the circuit diagram (see Figure 12) is provided. The task of the two force resistors was to detect the range of impact depending on the threshold set through the toggle switch (advanced - higher threshold, beginner lower threshold). Corresponding LED's would light up - for example with low impact the yellow LED's would be activated. Upon flicking the toggle switch, a timer was initiated at the end of which an audio clip was played to allow for sharing or turn taking. All audio clips were stored in the micro SD card which was connected to the microprocessor. Simple program logic determined which appropriate audio clip would be played depending on the game context. A disk vibrator motor was also used to provide haptic feedback in the handle upon impact.

A final evaluation session was carried out which comprised of a short observation session while children were playing with the GiggleBat followed by a focus group with their mothers who were the same seven mothers as the second focus group. The session took place in a park. The aim of the session 


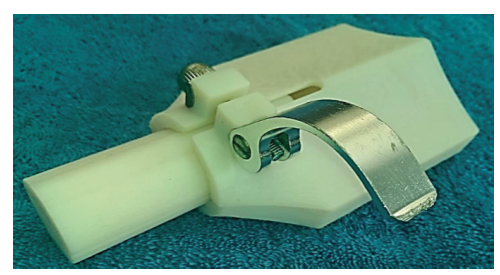

Figure 9: $3 D$ model of the proposed attachment mechanism

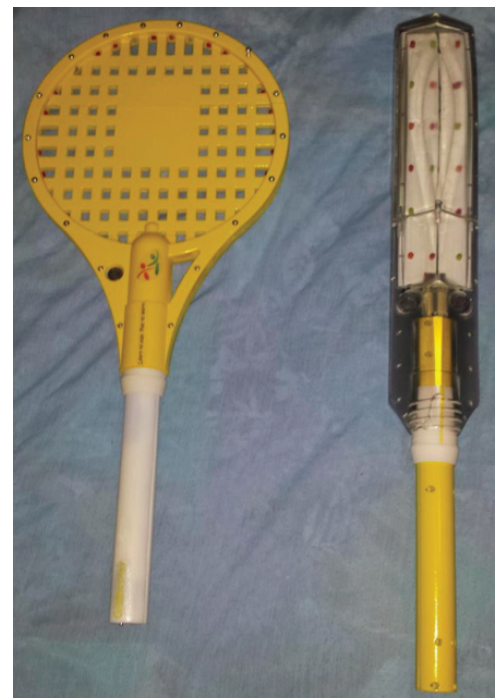

Figure 10: Final Prototypes

\begin{tabular}{|c|c|}
\hline Component & Function \\
\hline Arduino Micro & Runs the program coding. \\
\hline Powerboost $1000 \mathrm{c}$ & $\begin{array}{l}\text { Charges the battery by micro USB and increased } \\
\text { voltage to } 5 \text { volts. }\end{array}$ \\
\hline $3.7 v 18650$ battery & Powers the circuitry. \\
\hline Disk vibration motor & Vibrates in the handle if a near perfect hit is made. \\
\hline SD card reader & Reads the sound files from a Micro SD card. \\
\hline 3-way toggle switch & Selects between beginner, advanced and off positions. \\
\hline Amplifier & Increases the volume of the speakers. \\
\hline Speaker & Plays the sound files. \\
\hline LEDs & Lights up when impact is made. \\
\hline Force resistor & Detects different ranges of impact. \\
\hline
\end{tabular}

Figure 11: Electronic components of GiggleBat

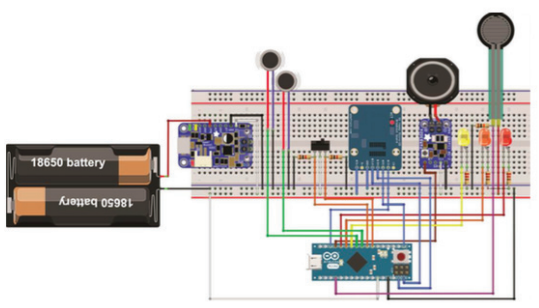

Figure 12: Circuit Sketch was for the mothers to physically view GiggleBat while their children tried it out in a short cricket match and then discuss its features, usability, impact, value, etc amongst other aspects in a focus group. Five children played with the bat for about 30 minutes in total. The bat would ask for the child to hand over the bat to the next child after 5 minutes. As researchers we took notes of both verbal and nonverbal behaviour from the children while playing.

The mothers appreciated the finalised concept and immediately stated that this would be a toy that would be very suitable for backyard cricket, beach cricket or park cricket (all of which are an integral component of Australian cricket culture) - particularly due to its portability and quick gameplay. The tennis racquet was also demoed but all the children wanted to try out the cricket bat. The tennis racquet incorporated a similar software to the cricket bat, however we acknowledge that the hitting dynamics of cricket and tennis are different. Our main purpose of implementing and prototyping the tennis racquet was to exhibit the possibility of the modular handle.

Despite a preference from the mothers for the clamp attachment mechanism in Focus Group 2 the children (all of whom were between 3 and 5) found it difficult to manipulate. In addition, the mothers recommended a rethink in strategy for the clamp attachment for two reasons: firstly, the force needed to clamp the handle was excessive for the target audience, and secondly, the general consensus was that the clamp was too bulky, and a more slimline, aesthetically pleasing approach would be more successful. The mothers appreciated the turn taking feature implemented through GiggleBat in particular and realised that playing with it would be highly entertaining for their children especially if the game could be quickly switched.

The representations of the voice feedback and lights were well received by the children as evidenced by their verbal behaviour during gameplay. The children were often seen replying to the bat, conducting a conversation-like sequence with the toy. The children specifically reacted with excitement on the targeted dialogue generated by the bat based on their shot impact. We had defined three impact thresholds each of which comprised of three set dialogue statements. During our session it was also observed that the children complied almost immediately with the instructions from GiggleBat to hand it over to the next child when their turn was over. Ultimately we are of the opinion that the turn taking feature can facilitate social development in preschool children. The thresholds of the force resistor applied to the difficulty levels seemingly suited the utilised play dimensions and ball type 
(simple tennis ball), however future research must investigate an adaption to playing area size (for example a backyard will be much smaller). With time, the children understood that the LED visualisations corresponded to their shot impact (red indicating strongest impact, followed by green and yellow). According to their verbal statements, most children appreciated the vibration in the handle as it reminded them of gaming consoles from home. Sustained and longitudinal evaluations of GiggleBat will be required to realise these aspects further. The children also wished to know whose shot generated the most impact and which player was the most successful. Three children also asked for humorous or audio clippings from cartoons as some of them did not immediately recognise the cricket commentators.

\section{CONCLUSION AND FUTURE WORK}

GiggleBat was designed through a focused attempt to include mothers and children in the design process to better target overlooked wants and needs. This was achieved through 3 focus groups at various stages throughout the design conceptualisation and design implementation. Ultimately GiggleBat limits the amount of sedentary activity, is simple to use, distinguishes scalability and is customisable to an extent. Promoting outdoor use and play, GiggleBat is well suited to Australian culture. In the future, we aim to continue with our development of GiggleBat and conduct further evaluations with children. Our first priority will be to carry out longitudinal evaluations with children to fully determine the efficacy, success, usability, entertainment value of our product.

We would now also like to reflect on the design methodology of our research. This project shows how industrial design and $\mathrm{HCl}$ together can make better products together, improve user participatory research and human experience and open up artifacts potential to multiple learning through software updates and tangible interaction. The new vision of Industrial Design is gradually overlapping with the interactive possibilities provided by $\mathrm{HCl}$ (Overbeeke and Hummels 2013). We were very fortunate to have a multidisciplinary team that could contribute across both domain areas. In our initial design process we have extensively focused on the contributions of mothers who play a key social partner of pre-school children (Prior and Glaser 2006). We conducted a final evaluation with children as "testers" (Druin 2002) of our prototype. Based on the qualitative feedback of children, we believe that they were positive about GiggleBat however a wow-factor or novelty effect must be acknowledged. Further longitudinal evaluations with GiggleBat can be expected to reveal additional insights from children. We also aim to involve the parents and mothers explicitly in the play activity even if they are not physically present - for example through audio snippets transmitted over a mobile connection when a particular event happens in the game.

We would also like to implement GiggleBat across other sports such as tennis and golf to fully realise the capability of the modular handle. We realise that the current handle requires different accessories to retrofit to. We are working on a new design which should miniaturise and relocate the technical package to the end of a cricket bat or tennis racket while keeping sensor terminals within a rubberised membrane sleeve. The sleeve could then be unrolled from the end onto any handle. Potential applications will be as many as handles are available since ergonomics for handles are similarly specific to children and adults. Through implementing $\mathrm{HCl}$ and communication into a sleeve or membrane that fits onto many sporting handles, GiggleBat can be effective for science and training concerns such as force, movement, technique, precision and coordination, while further implementation into the child market can be achieved through addressing other motivational factors.

\section{REFERENCES}

Anlauff, J., E. Weitnauer, A. Lehnhardt, S. Schirmer, S. Zehe, and K. Tonekaboni (2010). A method for outdoor skateboarding video games. In Proceedings of the 7th International Conference on Advances in Computer Entertainment Technology, pp. 40-44. ACM.

Attewell, P., J. Battle, and B. Suazo-Garcia (2003). Computers and young children: Social benefit or social problem? Social forces 82(1), 277-296.

Australia, C. (2015). Census results reveal cricket's changing face.

Avontuur, T., R. de Jong, E. Brink, Y. Florack, I. Soute, and P. Markopoulos (2014). Play it our way: customization of game rules in children's interactive outdoor games. In Proceedings of the 2014 conference on Interaction design and children, pp. 95-104. ACM.

Bekker, T., J. Sturm, R. Wesselink, B. Groenendaal, and B. Eggen (2008). Interactive play objects and the effects of open-ended play on social interaction and fun. In Proceedings of the 2008 International Conference on Advances in Computer Entertainment Technology, pp. 389392. ACM.

Bekker, T. M. and B. H. Eggen (2008). Designing for children's physical play. In CHI'O8 extended 
abstracts on Human factors in computing systems, pp. 2871-2876. ACM.

Bogdanovych, A. and C. Stanton (2015). A novel approach to sports oriented video games with real-time motion streaming. In Proceedings of the 7th ACM SIGCHI Symposium on Engineering Interactive Computing Systems, EICS '15, pp. 6473. ACM.

Cassell, J. and K. Ryokai (2001). Making space for voice: Technologies to support childrens fantasy and storytelling. Personal and ubiquitous computing 5(3), 169-190.

Chase, M. A. and M. Machida (2011). The role of sport as a social status determinant for children: Thirty years later. Research quarterly for exercise and sport 82(4), 731-739.

Daley, A. J. (2009). Can exergaming contribute to improving physical activity levels and health outcomes in children? Pediatrics 124(2), 763-771.

De Oliveira, R. and N. Oliver (2008). Triplebeat: enhancing exercise performance with persuasion. In Proceedings of the 10th international conference on Human computer interaction with mobile devices and services, pp. 255-264. ACM.

Dietz, W. H. and S. L. Gortmaker (2001). Preventing obesity in children and adolescents 1. Annual review of public health 22(1), 337-353.

Druin, A. (2002). The role of children in the design of new technology. Behaviour and information technology 21(1), 1-25.

Hendrix, K., G. Yang, D. van de Mortel, T. Tijs, and P. Markopoulos (2008). Designing a headup game for children. In Proceedings of the 22nd British $\mathrm{HCl}$ Group Annual Conference on People and Computers: Culture, Creativity, InteractionVolume 1, pp. 45-53. British Computer Society.

Henrique, R. S., A. H. Ré, D. F. Stodden, J. Fransen, C. M. Campos, D. R. Queiroz, and M. T. Cattuzzo (2015). Association between sports participation, motor competence and weight status: A longitudinal study. Journal of Science and Medicine in Sport.

Jensen, M. M., M. K. Rasmussen, and K. Grønbæk (2013). Exploring opponent formats. In Entertainment Computing-ICEC 2013, pp. 48-60. Springer.

Lu, F., F. Tian, Y. Jiang, X. Cao, W. Luo, G. Li, X. Zhang, G. Dai, and H. Wang (2011). Shadowstory: creative and collaborative digital storytelling inspired by cultural heritage. In Proceedings of the SIGCHI Conference on Human
Factors in Computing Systems, pp. 1919-1928. ACM.

MacLean, A., R. M. Young, V. M. Bellotti, and T. P. Moran (1991). Questions, options, and criteria: Elements of design space analysis. Humancomputer interaction 6(3-4), 201-250.

Mubin, O., D. Rutishauser, M. Novoa, D. Wainohu, and S. Shahid (2015). Interactive toys in the home: A parents perspective. In HumanComputer Interaction-INTERACT 2015, pp. 573577. Springer.

Mubin, O., L. Vink, P. Oosterwijk, A. Al Mahmud, and S. Shahid (2013). Floffy: Designing an outdoor robot for children. In HumanComputer Interaction-INTERACT 2013, pp. 563570. Springer.

Mueller, F., R. A. Khot, A. D. Chatham, S. Pijnappel, C. C. Toprak, and J. Marshall (2013). Hci with sports. In CHl'13 Extended Abstracts on Human Factors in Computing Systems, pp. 2509-2512. ACM.

Mueller, F. and M. Muirhead (2015). Jogging with a quadcopter. In Proceedings of the 33rd Annual ACM Conference on Human Factors in Computing Systems, pp. 2023-2032. ACM.

Mueller, F. F., J. Marshall, R. A. Khot, S. Nylander, and J. Tholander (2015). Understanding sportshci by going jogging at chi. In Proceedings of the 33rd Annual ACM Conference Extended Abstracts on Human Factors in Computing Systems, pp. 869-872. ACM.

Nitz, J., S. Kuys, R. Isles, and S. Fu (2010). Is the wii fit a new-generation tool for improving balance, health and well-being? a pilot study. Climacteric 13(5), 487-491.

of Health, D. and Ageing (2010). Children who are overweight or obese. Australian Bureau of Statistics 1301.

Olds, T. S., G. R. Tomkinson, K. E. Ferrar, and C. A. Maher (2010). Trends in the prevalence of childhood overweight and obesity in australia between 1985 and 2008. International journal of obesity 34(1), 57-66.

Overbeeke, K. and C. Hummels (2013). Industrial design. The Encyclopedia of Human-Computer Interaction, 2nd Ed..

Papert, S. and I. Harel (1991). Situating constructionism. Constructionism 36, 1-11.

Prior, V. and D. Glaser (2006). Understanding attachment and attachment disorders: Theory, evidence and practice. Jessica Kingsley Publishers. 
Putnam, R. D. (2001). Bowling alone: The collapse and revival of American community. Simon and Schuster.

Robins, B., E. Ferrari, and K. Dautenhahn (2008). Developing scenarios for robot assisted play. In Robot and Human Interactive Communication, 2008. RO-MAN 2008. The 17th IEEE International Symposium on, pp. 180-186. IEEE.

Rowe, D. (2013). Sport: scandal, gender and the nation. Institute for Culture and Society Occasional Paper Series 4(3), 1-17.

Soute, I., P. Markopoulos, and R. Magielse (2010). Head up games: combining the best of both worlds by merging traditional and digital play. Personal and Ubiquitous Computing 14(5), 435-444.

Sturm, J., R. Tieben, M. Deen, T. Bekker, and B. Schouten (2011). Playfit: Designing playful activity interventions for teenagers. In Proceedings of DIGRA, pp. 14-17.

Toering, E., I. Soute, and P. Markopoulos (2010). Rule customization in head-up games. In Proceedings of the 3rd International Conference on Fun and Games, pp. 144-148. ACM.

Toprak, C. C., J. Platt, and F. Mueller (2012). Bubble popper: considering body contact in games. In
Proceedings of the 4th International Conference on Fun and Games, pp. 97-100. ACM.

Toscos, T., A. Faber, S. An, and M. P. Gandhi (2006). Chick clique: persuasive technology to motivate teenage girls to exercise. In CHl'06 extended abstracts on Human factors in computing systems, pp. 1873-1878. ACM.

Vygotsky, L. S. and J. Wollock (1997). The Collected Works of LS Vygotsky: Problems of the theory and history of psychology, Volume 3. Springer Science \& Business Media.

Wake, M., P. Hardy, L. Canterford, M. Sawyer, and J. Carlin (2007). Overweight, obesity and girth of australian preschoolers: prevalence and socio-economic correlates. International journal of obesity 31(7), 1044-1051.

Wood, J. J., B. D. McLeod, M. Sigman, W.-C. Hwang, and B. C. Chu (2003). Parenting and childhood anxiety: Theory, empirical findings, and future directions. Journal of child psychology and psychiatry 44(1), 134-151.

Zakus, D., J. Skinner, and A. Edwards (2009). Social capital in australian sport. Sport in society 12(7), 986-998. 